\title{
Tsafon
}

Revue d'études juives du Nord

$80 \mid 2020$

Varia

\section{Rachel traductrice de poésie de langue française}

\section{Bernard Grasset}

\section{(2) OpenEdition}

Journals

Édition électronique

URL : https://journals.openedition.org/tsafon/3152

DOI : 10.4000/tsafon.3152

ISSN : 2609-6420

\section{Éditeur}

Association Jean-Marie Delmaire

Édition imprimée

Date de publication : 1 décembre 2020

Pagination : 37-65

ISSN : 1149-6630

\section{Référence électronique}

Bernard Grasset, «Rachel traductrice de poésie de langue française », Tsafon [En ligne], 80 | 2020, mis en ligne le 01 décembre 2020, consulté le 24 juin 2021. URL : http://journals.openedition.org/tsafon/ 3152 ; DOI : https://doi.org/10.4000/tsafon.3152 


\section{Rachel traductrice de poésie de langue française}

Bernard Grasset*

Rachel est connue comme poétesse, auteur de Regain (1927), De loin (1930) et Nébo (1932). Mais elle fut aussi l'auteur d'articles littéraires, pleins de vivacité, riches de vues singulières, et son chemin poétique est passé par un travail, important à ses yeux, de traduction, par un séjour au sein de langues très différentes. Ainsi a-t-elle traduit de l'hébreu au russe: Haïm-Nahman Bialik, Jacob Fichman et Zalman Schnéour lors de son retour de France en Russie, à la fin de la Première Guerre mondiale. Ce travail de traduction s'est poursuivi lors de sa seconde installation en Palestine, de 1919 à sa mort, en contrepoint de l'écriture de ses propres poèmes, comme si cette activité de traduction était inséparable de l'activité de création poétique. Ainsi a-t-elle traduit des poètes du russe à l'hébreu : Anna Akhmatova, Maria Morévskaia, Maria Shkapskaia, Alexandre Pouchkine, Innocent Annenski, Vladislav Khodasevich, Serge Essénine. De l'allemand à l'hébreu : Heinrich Heine. De l'anglais à l'hébreu : Jessie Sempter. De l'italien à l'hébreu : Ada Négri. Du yiddish à l'hébreu : Abraham Liesin. Du français à l'hébreu : Francis Jammes, Charles Van Lerberghe, Maurice Maeterlinck, Paul Verlaine. Plutôt que de traduire des œuvres entières, Rachel a préféré traduire des poèmes choisis et voyagé d'une langue à l'autre, entre les cultures. Seuls quelques-uns de ces poèmes furent publiés en revues, de son vivant, mais l'ensemble (une trentaine de pages) a été réuni dans l'édition de 1980, Poésie de Rachel (Shirat Rahel). On note parmi les langues traduites une nette dominante du français, à côté du russe : sur les vingt-trois poèmes traduits en hébreu par Rachel, huit l'ont été du

\footnotetext{
* Membre associé de l'Équipe de recherche «La Bible et ses lectures », Université catholique de l'Ouest (Angers) - UMR 8167, Orient \& Méditerranée.

Les poèmes et leur traduction sont publiés à la fin de l'article.
} 
français, soit un peu plus du tiers et sur les quinze auteurs de ces poèmes, quatre sont de langue française, soit près du quart. À partir de ce constat et de celui de l'importance de la traduction poétique pour l'acte de création poétique chez Rachel, il n'a pas paru sans intérêt d'étudier méthodiquement, précisément, son travail de traductrice de la langue française, de la poésie française.

\section{1 - Paul Verlaine}

Né le 30 mars 1844 à Metz, Paul Verlaine s'est éteint le 8 janvier 1896 à Paris (rue Descartes). Menant une vie d'une grande turbulence, il a composé une œuvre poétique d'une valeur inégale de laquelle se détachent les Poèmes saturniens (1866), Romances sans paroles (1874) et Sagesse (1880). Converti en 1874 pour quelques printemps, il sera élu Prince des poètes en 1894. Sans doute Rachel aimait-elle dans la poésie verlainienne ce qui en constitue la principale qualité, à savoir la musicalité. Le poème, en mode mineur, à mi-voix, se rapproche de la chanson, du chant. "Écoutez la chanson bien douce» - «Écoutez la chanson bien sage $»^{1}$. S'il peut y avoir un goût pour la simplicité, présent dans le poème de Verlaine qu'elle traduit ${ }^{2}$, Rachel ne mentionne pas ce poète comme cultivant la vertu de simplicité dans son article Sur le signe $d u$ temps où elle s'attache à mettre en évidence l'idéal esthétique d'union de la simplicité et de la force, pas plus d'ailleurs que Van Lerberghe et Maeterlinck dont elle traduit aussi des poèmes ${ }^{3}$. La poésie mélancolique, $\mathrm{du}$ souvenir, de l'auteur des Poèmes saturniens ${ }^{4}$ pouvait rejoindre 1'auteur de Regain. En ce qui concerne Sagesse, d'où est extrait le poème traduit par Rachel, ce recueil est paru en décembre 1880 à compte d'auteur et visait à mettre en œuvre, avec sincérité, un art poétique catholique. Si Rachel l'a lu en entier, elle a dû être sensible au sentiment de la mort, au poème devenant chanson, à la chanson devenant oraison, à l'amour déchiré de Dieu, aux variations du cœur, à des accents mystiques ${ }^{5}$.

\footnotetext{
${ }^{1}$ Tels sont le premier et le dernier vers du poème I, XVI de Sagesse. Au recueil qu'il fait imprimer en 1870, Verlaine donne le titre de La Bonne Chanson.

${ }^{2}$ « [...] la vie est là / Simple et tranquille », (Sagesse, III, VI). Voir aussi ibid., I, XVI : « être simple sans plus attendre ».

${ }^{3}$ En revanche elle cite Paul Fort, Charles Vildrac, Francis Jammes. Voir Tsafon, $\mathrm{n}^{\mathrm{o}} 78$, automne 2019-hiver 2020, p. 109-124.

${ }^{4}$ Voir dans ce recueil Nevermore, Chanson d'automne.

${ }^{5}$ «Ô mon Dieu, vous m'avez blessé d'amour »- « Mais ce que j'ai, mon Dieu, je vous le donne » : ainsi commence, ainsi s'achève le poème II, I de Sagesse. «La paix du
} 
Rachel n'a traduit qu'un seul poème de Paul Verlaine mais sans doute le plus beau. Qu'est-ce qui a pu attirer Rachel dans Le ciel est pardessus le toit, ce texte écrit en septembre 1873 (après la rupture violente avec Rimbaud), comme l'acmé du recueil Sagesse paru en 1880 et qui signe poétiquement la conversion (provisoire) de Verlaine ? On pense à la musicalité du poème, sa proximité avec la prière, le goût pour ce qui est simple et calme, la sensibilité à la nature et le caractère poignant, déchirant, de la conclusion. Quel art de traduire Rachel met-elle en œuvre ? Sans renoncer au sens littéral, elle privilégie le sens poétique. Ainsi cherche-t-elle à faire en sorte que le poème en hébreu soit véritablement un poème, quitte à ajouter ou supprimer des mots, modifier l'ordre. La couleur bleue [tekhèlét] ${ }^{6}$ qui apparaît au second vers du poème de Verlaine en parallèle au mot «calme » devient le premier mot du poème en hébreu et se trouve ainsi soulignée ${ }^{7}$. Au troisième vers, Rachel ajoute le mot « cime» devant « arbre ». Dans le quatrième vers disparaît de l'hébreu le mot "palme". Dans le second quatrain le «Doucement tinte» verlainien se trouve traduit par «S'élève silencieusement». À la fin du quatrième quatrain, Rachel ajoute « et des hommes» après «de la ville» afin, sans doute, de trouver une rime. Dans le dernier quatrain, les « pleurs » sont qualifiés d' « amers » tandis que disparaît le « sans cesse ». Rachel, qui s'attache à donner une version rimée du poème de Verlaine, se montre traductrice à la fois fidèle et libre.

\section{2 - Maurice Maeterlinck}

Maurice Maeterlinck, sur lequel Rachel a écrit par ailleurs son plus long article La Vie des Fourmis, est né à Gand en 1862. Docteur en droit, il a traduit le Livre des XII béguines et L'Ornement des noces spirituelles de Ruysbroeck l'Admirable $(1885,1891)$ ainsi que Les Disciples à Saïs et les Fragments de Novalis (1895). Lors de l'affaire Dreyfus, il a soutenu ce dernier, à la suite de son ami Charles Van Lerberghe. En

cœur, l'amour d'être pauvre, et mes [Dieu] soirs // Mystiques, quand l'esprit s'ouvre aux calmes espoirs » (Sagesse, II, IV, 7). Par ailleurs le vers conclusif de Sagesse, I, XI, "Le seul savant, c'est encore Moïse », ne pouvait que toucher Rachel.

${ }_{7}^{6}$ C'est la couleur de prédilection de Rachel, cette couleur qu'elle associe à Tibériade.

${ }^{7}$ La poésie verlainienne aime revenir au bleu du ciel : «- Qu'il était bleu, le ciel, et grand, l'espoir !» (Colloque sentimental, Fêtes galantes); «Le ciel tout bleu, comme une haute tente » (XIX, La Bonne Chanson) ; «Le ciel tout bleu (I, VII, Sagesse) et « Le ciel (...) / Si bleu » (poème traduit). 
1911, l'auteur de Pelléas et Mélisande reçoit le Prix Nobel de littérature. Il s'est éteint en 1949 à Nice. Attiré à la fois par la science et la poésie, cultivant esprit scientifique et esprit poétique, il était aussi passionné par la botanique ${ }^{8}$, la vie des insectes, tout en aimant pratiquer le sport. Poètepenseur, en recherche des clartés intérieures, outre des œuvres théâtrales significatives comme La Princesse Maleine (1889), il publie des essais de nature philosophique comme Le Trésor des humbles (1896) ou Sagesse et destinée (1898), le recueil de Serres chaudes (1889), remarqué par les surréalistes. Attentif à la poésie populaire, à ses qualités musicales, il publie Douze Chansons en 1896 qui deviendront Quinze Chansons en 1900. C'est le plus beau poème et le plus connu de ce recueil, Et s'il revenait vers nous..., que Rachel choisit de traduire en hébreu.

Comme Rachel, Maeterlinck, qui affirmait : « J'ai toujours agi, j'ai toujours pensé comme si j'eusse dû m'en aller demain $»^{9}$, avait une conscience aiguë de sa mortalité. Énigme suprême, la mort marquerait notre pauvreté extrême ${ }^{10}$. Maeterlinck élevait un mur de silence protecteur autour de lui ${ }^{11}$. À la confluence du monde germanique et du monde latin, sa pensée, aspirant à la lumière et à l'amour, comporte une dimension spiritualiste et humaniste. Il se fixe comme devoir de réduire le mal et d'augmenter la fraternité. En revanche, il estime, ce qui est discutable et qui n'est pas la position de l'auteur de Regain, que l'esthétique doit se dissocier de l'éthique. L'écriture de Maeterlinck est remplie de symboles mais qui ne sont pas aussi imprégnés de la Bible que ceux de la poésie de Rachel. Monde symbolique, c'est aussi un monde de rêves où l'étrange se surimprime sur le réel. «Seigneur, les rêves de la terre / Mourront-ils enfin dans mon cœur !» (Serres chaudes, Tentations). Poésie du symbole, du rêve, marquée par l'inconnu qui ne cesse de nous entourer, poésie qui se fait à l'occasion supplique, l'écriture de Maeterlinck reste hospitalière à l'infini. Il n'est de « beauté $[\ldots]$ vaste et $[\ldots]$ profonde $»$ sans $«$ quelque chose d'infini $»^{12}$. Ainsi en

\footnotetext{
${ }^{8}$ Dans les lettres que Rachel écrit de France en 1913 à Samuel Dayan et à Noé Naftolsky, elle exprime son enthousiasme pour l'étude de la botanique, du secret des plantes et, plus largement, du «mystère des mystères de la vie de toute la nature ».

${ }^{9}$ Cité dans Roger Bodart, Maurice Maeterlinck, Paris, Seghers, Poètes d'aujourd'hui, 1962, p. 41.

${ }^{10}$ Serres chaudes, Quinze chansons, La Princesse Maleine, Paris, Poésie / Gallimard, 2016, La Princesse Maleine, V, p. 268.

${ }^{11}$ Rachel évoque dans son poème Mur (De loin) «Le mur» qui la «sépare / Des autres ».

${ }^{12}$ Préface à l'édition du Théâtre de 1901, dans Serres chaudes..., op. cit., VII, p. 300.
} 
est-il de la poésie de Rachel qui accorde par ailleurs une place significative à l'attente. " Il faut attendre, il faut attendre, / Il faut attendre d'autres jours... » (Quinze Chansons, XII).

De même que pour Verlaine, Rachel n'a traduit qu'un seul poème de Maurice Maeterlinck. Un poème dont la brièveté des vers, la densité, la simplicité mêlée de profondeur, la proximité avec la chanson ont dû l'attirer. Alors que le poème n'a pas de titre en français, Rachel lui a donné le titre de zémér ${ }^{13}$, chant, évoquant par là le titre du recueil de Maeterlinck dont il est tiré : Quinze chansons. En imitant le texte français où les deuxième et quatrième vers de chaque quatrain riment, elle parvient, non sans virtuosité, à faire aussi rimer ces vers en hébreu. Elle renonce à reprendre les points de suspension qui concluent les quatrains de Maeterlinck. À la fin du premier quatrain, elle traduit «Jusqu'à s'en mourir... » par "Jusqu'à rendre l'âme » (la fin du mot âme [neshamah] rime avec le $[\mathrm{mah}]$ interrogatif du second vers). Le "Il souffre peutêtre» du second quatrain devient en hébreu "Peut-être son âme ${ }^{14}$ [néfésh] est amère ${ }^{15} »$. Dans le troisième quatrain disparaît $l^{\prime}$ « anneau d'or », apparaît «j'ai réclamé ». L'ajout de l'adjectif «froide» pour qualifier la salle au second vers et la traduction d' « ouverte » par « non fermée » pour qualifier la porte au quatrième vers semblent dus à la volonté de rimes. Dans le dernier quatrain le mot « heure » (au pluriel en hébreu) se trouve déplacé au premier vers tandis que dans le dernier vers Rachel ajoute «qu'il ne veuille» avant «pleurer». La poétesse ne s'enferme donc pas dans la lettre, travaille le rythme, la musique. Elle respecte le sens mais entend recréer un vrai poème en hébreu.

\section{3 - Charles Van Lerberghe}

Charles Van Lerberghe est né le 21 octobre 1861 à Gand, comme son ami Maurice Maeterlinck. Docteur en lettres et philosophie en 1894, il fait paraître deux recueils de poésie, Entrevisions en 1898 et La Chanson d'Ève en 1904. Ému par l'article J'accuse, il avait créé un comité Zola, défenseur de Dreyfus. Il meurt le 26 octobre 1907 à Bruxelles. Alors que Maeterlinck, juriste de formation, a publié des

\footnotetext{
${ }^{13}$ Chant, de la racine $z m r$ chanter mais aussi tailler (la vigne).

${ }^{14} \mathrm{Ou}$ : sa vie.

${ }^{15} \mathrm{Ou}$ : triste.
} 
essais philosophiques à côté de ses œuvres poétiques et théâtrales, Van Lerberghe, philosophe de formation, a renoncé à la philosophie au profit de la seule poésie ${ }^{16}$. La vérité, ce n'est pas dans la science, dans la pensée philosophique qu'il la trouve, mais dans la beauté, son éclat. La poésie de Charles Van Lerberghe n'a cessé de subsumer le réel dans le rêve, le visible dans l'invisible, la matière dans l'esprit. La vraie poésie, poésie des secrets de l'âme, ne se sépare pas d'un universel et constant symbolisme. Recouverte du voile du rêve, la terre émerveille le regard.

La poésie de Van Lerberghe, plus radicalement symbolique encore que celle de Maeterlinck, demeure, comme celle-ci, attentive au mystère et prend à l'occasion une coloration mystique. Nulle beauté, nulle vérité, sans hospitalité du mystère. «Un poème ne me plaît tout à fait que lorsqu'il est à la fois d'une beauté pure, intense et mystérieuse ${ }^{17}$. Le mystère s'éprouve par-delà l'apparence. «En mes yeux éblouis entre seul, Invisible ! / À mon oreille chante seul, Inouï ! ${ }^{18}$. Et si au fond n'existait vraiment, pleinement, que ce qui ne peut être ni entendu, ni vu. Et si le mystère était le divin. «Ô Dieu, qui donc est là, / Dans le vide, au-delà / de cette porte ! ${ }^{19}$. La poésie est appelée à devenir chanson des profondeurs, chant mystérieux.

«Le plus beau jardin calme, endormi / Dans le silence, dans le mystère ${ }^{20}$. Le mot «calme » est un leitmotiv de la poésie de Charles Van Lerberghe qui est en quête d'apaisement. Le calme conjoint à la douceur discerne le sacré. «Tout ce qui vient du ciel est béni $»^{21}$. Le regard apaisé découvre le bleu paradis. Il est « un autre monde », « une autre lumière », « un autre monde de beauté ». Ailleurs, au-delà de l'immédiat, du réel quotidien. Il reste au poète à vivre en attente, à guetter l'aube lointaine, percevoir l'infini murmure. Charles Van Lerberghe est poète de l'aube ${ }^{22}$. Sans doute est-ce tous ces thèmes qui ont

\footnotetext{
${ }^{16}$ « La philosophie que j'ai étudiée, que j'adore partout où elle s'harmonise avec la science, et ne cherche pas à la contredire, n'a cependant jamais pris en mes prédilections la place de la Poésie pure et de l'Art. Je mets la Poésie et l'Art au-dessus de tout ». Charles Van Lerberghe, Lettre du 4 août 1904 à Émile Lecomte, cité dans Hubert Juin, Charles Van Lerberghe, Paris, Seghers, Poètes d'aujourd'hui, 1969, p. 75.

${ }^{17}$ Ibid., p. 88. «Je crois aussi que toute profonde beauté est mystère, et que ce côté mystérieux est un signe qu'on l'a entrevue ».

${ }^{18}$ La Chanson d'Ève, Paris, Mercure de France, 1952 (1 ${ }^{\text {re }}$ éd. 1904), p. 74.

${ }^{19}$ Ibid., p. 140. On rapprochera ces vers du poème La porte s'ouvre (De loin) de Rachel. (De loin suivi de Nébo, trad. : Bernard Grasset, Paris - Orbey, Arfuyen, 2013, p. 97).

${ }^{20}$ Entrevisions, Bruxelles, Nouvelle Société d'Éditions, 1936, À l’insu, p. 65.

${ }^{21}$ La Chanson d'Ève, Ma sæur la pluie, p. 51.

${ }^{22} \mathrm{~L}$ 'aube ou l'aurore constituent un constant refrain de sa poésie. L'antépénultième vers du recueil Entrevisions évoque le chant dans l'aurore.
} 
dû attirer Rachel dans la poésie de ce poète belge. Ayant traduit trois poèmes de La Chanson d'Ève, elle avait dû lire l'ensemble de ce recueil dont le titre devait parler à son cœur de lectrice juive. Sans doute rejetaitelle les mièvreries, les facilités, les inconsistances de Van Lerberghe tout en appréciant sa poésie des symboles, du mystère, du rêve, le poème qui se fait chant, chanson des origines et de l'espérance.

De ce poète qui n'était pas le plus connu des poètes d'expression française au début du $\mathrm{XX}^{\mathrm{e}}$ siècle et qui est tombé dans l'oubli, Rachel a traduit trois poèmes. C'est donc l'un des poètes qu'elle a le plus traduits. Dans le poème Ma sœur la Pluie, c'est certainement le sens de la nature qui s'y exprime tout au long qui l'a retenue mais aussi le caractère à la fois pictural et musical des vers. Quelques remarques sur la traduction de Rachel, ses choix. Le mot «pluie » [matar], mot-clef du poème, est du genre masculin en hébreu d'où la traduction par "mon frère " ['ahi] au lieu de «ma sœur». Au début de la seconde strophe, Rachel inverse l'ordre des deux premiers vers. Plus loin, les pies disparaissent comme le chant des nids tandis qu'à la première occurrence du verbe "danser » Rachel préfère le verbe «jubiler». Elle traduit littéralement le dernier vers : «Tout ce qui vient du ciel est béni» dans lequel elle devait reconnaître une affirmation essentielle de sa propre poésie ${ }^{23}$. Au début de la strophe suivante, à la différence de l'original, elle répète le mot « lèvres », sans doute pour des raisons de rythme. Les « fleurs" ne sont plus qualifiées de « sonores ». Dans la dernière strophe, Rachel remplace dans le premier vers le verbe " essuyer", à propos de l'action du soleil, par « briller» tout en déplaçant « essuyer » à l'ultime vers. Nulle volonté donc de respecter coûte que coûte la lettre mais une aspiration à traduire librement la beauté d'un chant avec lequel la poétesse se sent en connivence.

Ne suis-je vous, n'êtes-vous pas moi est le second poème de Charles Van Lerberghe que traduit Rachel. Il est extrait de La Chanson d'Ève, parue en 1904. Dans le choix de ce poème, l'auteur de Regain a dû être guidée par l'évocation des fleurs et de la lumière, la communion avec la vie, l'interrogation sur l'existence, la présence du cœur, le style clair, rapide, fluide, la conclusion avec le rêve. Dans la traduction du

\footnotetext{
${ }^{23}$ « Tout est béni ! // Tout est béni, pour tout il est un chant consolateur » écrit-elle dans le poème Liesse fugace, daté de 1926, de Regain, trad. : Bernard Grasset, Paris - Orbey, Arfuyen, 2006, p. 61.
} 
premier vers, elle inverse l'ordre des deux stiques. Elle apporte une légère nuance en traduisant « de mes doigts » par « de ma main ». Tandis que, dans le texte de Charles Van Lerberghe, les huit premiers vers constituent une seule strophe, Rachel en fait deux strophes de quatre vers. Le « Âme qui penses » du sixième vers se trouve traduit par «Pensée de l'âme» placé au début du cinquième vers tandis que la traduction de « soleil où je luis » est déplacée au sixième vers. Rachel respecte l'enjambement entre le dixième et le onzième vers (mais non entre le neuvième et le dixième). Elle inverse volontairement les douzième et treizième vers et $\mathrm{y}$ traduit «beau fleuve» par «grand torrent ». L'ensemble de la traduction reste fidèle tout en jouant librement sur l'ordre des mots, des vers.

Troisième et dernier poème de Charles Van Lerberghe traduit par Rachel, le plus long: Quand vient le soir, est toujours extrait de La Chanson d'Ève. Dans la première strophe du texte original, la traductrice apporte quelques nuances, voire des variations, comme la traduction de «sortent» par «montent», celle du dernier vers sous le mode interrogatif et l'effacement de l'adjectif possessif de la première personne pluriel au profit du simple article défini pluriel. Des deux premières strophes du poème de Van Lerberghe, Rachel fait une seule et unique strophe. Au début de la seconde strophe du texte original, Rachel souligne le simple « crépuscule » en le précisant par « pénombre ». Plus loin le verbe «s'appeler» est traduit par «se rassembler» tandis que le «sans bruit» disparaît. Dans la troisième strophe du poème de Van Lerberghe, Rachel remplace l'eau par le sommeil et traduit «grand flambeau » par « flambeau splendide ». Le « En rêve » n'est pas traduit et les "yeux bleus » deviennent, par nuance, dans le dernier vers, les « yeux de saphir » ce qui permet une rime avec le troisième vers. Dans la dernière strophe du poème, le quatrième vers évoquant l'eau, la terre, se trouve traduit par deux vers mais comme Rachel ne traduit pas le sixième vers, le nombre de vers de la traduction reste le même. Le « Enfin » n'est pas traduit et le «Et c'est matin » conclusif est rendu sobrement par « Le matin vient $»^{24}$. Rachel use de temps à autre de rimes sans souci de se conformer strictement à la lettre du texte original où elles sont plus nombreuses. Elle a dû aimer dans ce poème l'expression, à partir de la nature, du temps qui passe, la part donnée au symbolique, au rêve, une douce interrogation sur le secret de l'univers.

\footnotetext{
${ }^{24} \mathrm{Ou}$ : est venu. $B a^{\prime}$ en hébreu peut signifier à la fois le présent ou le passé, $3^{\mathrm{e}}$ pers. masc. sing.
} 


\section{Francis Jammes}

Très présent dans l'œuvre et dans le cœur de Rachel, Francis Jammes se révèle défenseur d'un art de la simplicité et en harmonie avec la nature. Né à Tournay (Hautes-Pyrénées) et mort à Hasparren (Pyrénées-Atlantiques), il a non seulement le goût de la nature mais aussi celui de l'enfance, de la vie humble. Après avoir longtemps vécu à Orthez, Jammes s'installe à Hasparren en 1921. Père de sept enfants, il mène une existence rurale, imprégnée de simplicité franciscaine, tout en entretenant une riche correspondance avec des auteurs comme Mallarmé, Gide, Samain, Claudel. Jusqu'à sa mort, il écrit, publie, poésies, romans. Ce poète singulier a été admiré hors de France par des auteurs aussi marquants que Kafka et Rilke. Parmi ses œuvres poétiques, on peut citer De l'Angélus de l'aube à l'Angélus du soir (1898), Le Deuil des primevères (1901) et, après sa conversion, Clairières dans le ciel (1906), Les Géorgiques chrétiennes (1911-1912), Les Livres des Quatrains (1923-1925).

C'est sans doute à l'occasion de son séjour à Toulouse, à partir de 1913 afin d'y étudier l'agronomie au sein de l'Université, que Rachel découvre, à l'âge de 23 ans, l'œuvre de Francis Jammes, qui vivait dans des terres presque voisines, et le jammisme, en même temps qu'elle a pu avoir un contact concret et direct avec la langue française. L'attachement de la poétesse de Tibériade au poète bucolique, né vingt-deux ans avant elle et mort sept ans après elle, s'est manifesté sous trois aspects : la citation de son nom dans le quatrième vers du premier quatrain de son poème Qui suis-je?, écrit à Safed en 1925 et qui fait partie de son premier recueil, Regain $^{25}$; la traduction de trois poèmes ${ }^{26}$ et l'écriture d'un article $^{27}$. La nature, les animaux et la simplicité, l'antiintellectualisme, la vie humble et l'attrait pour l'infini, un lyrisme voilé de nostalgie, voilà tout ce qui chez ce poète, converti au catholicisme en

\footnotetext{
${ }^{25}$ Écoutons Rachel qui, après s'être définie comme « calme », à l'image d'un lac, dans les deux premiers vers du poème, écrit : «J'aime la sérénité de l'attente, les yeux des enfants / Et les poèmes de Francis Jammes ». Ainsi proclame-t-elle son amour pour la poésie du poète français. Il convient de souligner que, dans toute l'œuvre poétique de Rachel qui comprend 144 poèmes en hébreu, répartis en trois recueils, Regain, De loin et Nébo, ou encore épars à sa mort, aucun autre nom de poète ne se trouve cité. Signe clair, évident, incontestable, de son sentiment de profonde proximité avec Jammes.

${ }^{26}$ Alors que généralement elle ne traduit qu'un seul poème par auteur.

${ }^{27}$ Jammes apparaît également dans un autre article, Signe du temps. C'est ainsi le seul auteur dont le nom figure dans deux articles.
} 
1905, a pu rejoindre intérieurement l'aventure créatrice et existentielle de Rachel, artiste et paysanne.

Rachel a ainsi traduit trois poèmes de Francis Jammes. Le premier, le plus long de tous les poèmes qu'elle a traduits en hébreu et qui dépassent assez rarement une page, est Prière pour aller au Paradis avec les ânes. Ce poème, le plus difficile à traduire en hébreu, tant sur le plan syntaxique que sur le plan lexical, des huit poèmes en français appartient aux Quatorze prières qui concluent le recueil Le Deuil des primevères, paru en 1901. La présence de la nature, avec «la campagne en fête », le symbole du «chemin» si omniprésent dans la poésie de l'auteur de Regain, la proximité avec le monde vivant, l'humilité et la pauvreté, le poète ami des ânes, ces porteurs de fardeaux, le poème qui devient prière, « la paix » et le « ciel bleu », tout cela parlait au cœur créateur de Rachel. Dans sa traduction, Rachel ramène le titre à un seul mot: Prière [Tephillah]. Tandis que la première strophe est composée de douze vers en français, elle la décompose en quatre strophes qui totalisent treize vers. Pour traduire le verbe "poudroyer», elle ajoute le substantif "poussière", sujet du verbe "s'élever». Au quatrième vers du texte français, elle ajoute le qualificatif de «droit» à « chemin ». Trois [waw] « et », caractéristiques de la langue hébraïque qui, langue de parataxe et non d'hypotaxe, les multiplie, sont ajoutés en début de vers. Rachel supprime deux adjectifs : « doux » devant « amis » et « pauvres » devant «bêtes ». En ce qui concerne la seconde strophe du texte français, composé de vingt-et-un vers, Rachel la traduit en dix-neuf vers répartis sur quatre strophes (dont l'une a un seul vers). Au premier vers, elle ajoute «mon Dieu» [Éli]. Au second vers, le «que j'aime tant» de Jammes est traduit par «d'un amour passionné ». Les vers sept à neuf évoquant les voitures de saltimbanques, de plumeaux ou de fer-blanc et les bidons, se trouvent traduits par un seul et unique vers en hébreu qui synthétise ici fortement en distinguant une seule catégorie de voitures ['agalot]. Plus loin les plaies «suintantes» deviennent, le sens étant intensifié, « ruisselantes de sang ». Au vers seize, l'adjectif «touffus» est supprimé tandis qu'au vers suivant Rachel ajoute «vierge» [betoulah] pour caractériser la jeune fille. L'adjectif « divines » du vers dix-huit se trouve rendu par le substantif complément «d'Éden » (soit édéniques). Enfin au dernier vers la «limpidité » est traduite, légère nuance, par «pureté ». La plus grande liberté prise par Rachel est la 
suppression de deux vers, l'ajout d'un vers dans la première strophe n'étant en réalité que la décomposition d'un vers en français en deux vers dont l'un devient le début d'une strophe en hébreu. Si nous avons par ailleurs pointé des nuances, des suppressions, des ajouts de mots, il convient de souligner, pour conclure, la grande maîtrise à la fois du français et de l'hébreu dont fait preuve Rachel pour traduire ce texte, à la langue difficile, de Jammes (le fait que celle-là qualifie la poésie de celui-ci de simple n'exclut pas la richesse de sa langue, ses subtiles variations et l'originalité de sa syntaxe).

Second poème de Jammes traduit par Rachel : Il va neiger... En réalité, ce poème, extrait de De l'Angélus de l'aube à l'Angélus du soir, publié en 1898, comporte sept quatrains mais elle choisit, sans doute pour des raisons esthétiques, de ne traduire que le premier de ces quatrains. Dans les quatre premiers vers du poème, la nature, le temps qui passe, le souvenir, la mélancolie, la solitude sont des thèmes que l'on retrouve, déclinés sous un autre mode, au travers de la poésie de Rachel. Celle-ci traduit simplement par "bientôt» au premier vers l'expression «dans quelques jours ». Au second vers elle ajoute la position assise près du feu et «qui a passé » après «ma tristesse ». Le vers, ainsi légèrement modifié, constitue en hébreu un parallélisme entre deux stiques, ordonnés l'un autour du feu, l'autre autour de la tristesse. Rachel inverse enfin l'ordre des troisième et quatrième vers ce qui lui permet de respecter scrupuleusement, avec virtuosité, l'aspect rimé du texte original.

Avec La salle à manger s'achève la traduction par Rachel des poèmes de Francis Jammes. Cette fois-ci elle traduit l'intégralité du poème qui, comme le précédent, appartient au recueil emblématique du poète pyrénéen, De l'Angélus de l'aube à l'Angélus du soir. Le dernier poème, La salle à manger, tout empreint de simplicité, évoque la mémoire de « la voix » du père, de « la voix » du grand-père, la présence invisible des ancêtres familiaux tandis que le style est marqué par l'anaphore du présentatif «Il y a ». Au niveau stylistique, on peut noter que le « il y a », [yèsh] en hébreu, sera une caractéristique de la poésie de Rachel où il est omniprésent. Quant au fond, elle se montrera très sensible à la présence secrète et silencieuse des morts qui l'accompagnent sur le chemin tragique de la vie ${ }^{28}$. Alors que la première strophe est composée de sept vers en français, Rachel sépare les quatre

\footnotetext{
${ }^{28}$ Ainsi en témoigne, de manière bouleversante, son dernier poème, Mes morts (Nébo), retrouvé sur la table de sa chambre après sa disparition. Voir Rachel, De loin suivi de Nébo, op. cit., p. 184-185 et note, p. 202.
} 
premiers vers, dont elle fait une strophe introductive se concluant par l'évocation de la voix paternelle, des trois suivants. Pour le reste, elle respecte les autres strophes de Jammes. On rencontre quelques rimes en hébreu mais sans volonté de reprendre l'ensemble des rimes (ou assonances) du poème en français. Dans les premiers vers du poème les trois relatives, «qui a... », caractérisant l'armoire, sont remplacées par de simples indépendantes, " elle a... », ce qui donne au texte de Rachel un autre charme, non moins réel. Au début de la seconde strophe du poème en français, le «coucou» est traduit par «horloge». Au cinquième vers, la voix brisée devient aussi cachée chez Rachel. Au troisième vers de la troisième strophe, l'ordre des substantifs (viande, pain et poires) est modifié, ce qui permet d'introduire une rime avec le dernier vers. À la fin de cette strophe, «serviteur fidèle» se trouve traduit par «serviteur dévoué » et la relative remplacée par une indépendante. Enfin dans la dernière strophe, pour le mouvement de « venir chez», Rachel use de «descendre chez», elle remplace « vivant» par « ici » et traduit par une périphrase « en cheminant vers le seuil» le «en entrant» du poème original. Au sein du dernier vers le tutoiement remplace le vouvoiement. De tous les poèmes écrits en français traduits par Rachel, c'est sans doute La salle à manger qu'elle traduit avec le plus de fidélité à la lettre.

Rachel a ainsi traduit quatre poètes de langue française, dont deux Belges. Si elle traduit un seul poème, choisi avec soin, pour Paul Verlaine et Maurice Maeterlinck, elle traduit trois poèmes pour Charles Van Lerberghe et Francis Jammes, extraits d'un même recueil pour le premier, de deux recueils différents pour le second. Le choix des poèmes de poètes de langue française effectué par Rachel traductrice nous révèle trois choses : l'ampleur de sa connaissance de la poésie francophone et son intérêt pour elle; la proximité que devait avoir le poème avec le chant $^{29}$ et la prière; l'importance du symbolisme dans son idéal esthétique $^{30}$. Si l'acméisme a joué un rôle non négligeable dans l'aventure poétique de $\operatorname{Rachel}^{31}$, on ne peut ignorer, et son travail de

\footnotetext{
${ }^{29}$ Notons à ce sujet que le mot [shir] en hébreu signifie à la fois poème et chant.

${ }^{30}$ Maeterlinck et Van Lerberghe ont écrit une œuvre sous le sceau du symbolisme. La poésie de Verlaine est, elle aussi, marquée par le symbolisme.

${ }^{31}$ L'acméisme était une école littéraire attachée à la concision, au concret et à l'héroïsme, au travail artisanal sur le langage. Parmi ses figures marquantes apparaissent
} 
traductrice le montre, que l'écriture symbolique constituait une forme de modèle pour elle.

Si l'on voulait caractériser en quelques mots, à la lumière de nos précédentes remarques, l'art de traduire de Rachel qui n'a pas craint d'affronter des textes, des vers, d'une redoutable difficulté, on le qualifierait d'art de la fidélité et de la liberté. On pourrait juger certaines des libertés qu'elle s'accorde en apparence un peu trop grandes mais il convient de souligner que la fidélité domine. La langue hébraïque et la langue française sont très différentes : l'une préfère coordonner et l'autre subordonner, l'une est pauvre en adjectifs qu'elle remplace par des noms à l'état construit, l'autre puise ses ressources lexicales dans l'héritage gréco-latin, l'une synthétise, l'autre analyse. Passer du français à l'hébreu n'est ainsi pas chose aisée. Rachel, qui choisit les poèmes qu'elle traduit avec une réelle sûreté de goût, y parvient avec un vrai talent de telle sorte que le poème traduit en hébreu demeure toujours un poème. On ne peut qu'admirer sa grande maîtrise de la langue française comme celle de la langue hébraïque (pourtant apprise tardivement). Artiste du langage, elle a aimé la culture française, elle a aussi aimé la langue française et l'a comprise jusqu'à la traduire. Si les articles de Rachel nous révèlent une authentique critique littéraire à côté de la noble poétesse que l'on connaissait, ses traductions poétiques nous font découvrir aussi une originale et rare traductrice ${ }^{32}$.

deux poètes russes : Anna Akhmatova dont Rachel a traduit quatre poèmes et Alexandre Blok.

${ }^{32}$ Un troisième volume consacré à Rachel (poèmes épars, articles, extraits de sa correspondance) doit paraître à l'automne 2021, dans notre traduction, chez Arfuyen. 


\section{1 - Paul Verlaine}

Le ciel est, par-dessus le toit,

Si bleu, si calme!

Un arbre, par-dessus le toit,

Berce sa palme.

La cloche, dans le ciel qu'on voit,

Doucement tinte.

Un oiseau sur l'arbre qu'on voit

Chante sa plainte.

Mon Dieu, mon Dieu, la vie est là

Simple et tranquille.

Cette paisible rumeur-là

Vient de la ville.

Qu'as-tu fait, ô toi que voilà

Pleurant sans cesse,

Dis, qu'as-tu fait, toi que voilà,

De ta jeunesse ${ }^{33}$.

${ }^{33}$ Extrait de Sagesse, 1880, III, VI. 


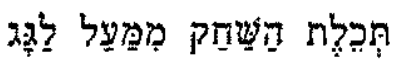

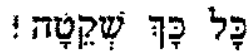

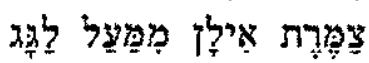

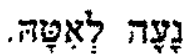

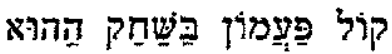

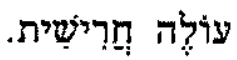

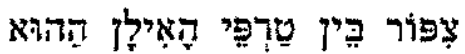

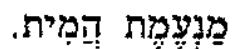

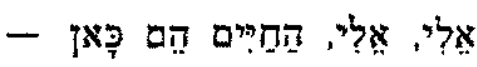

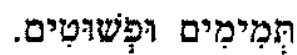

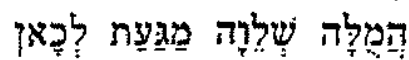

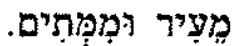

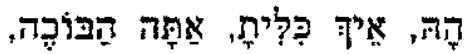

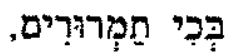

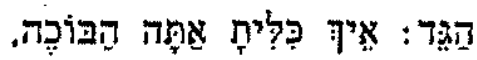

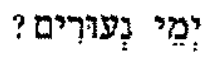




\section{2 - Maurice Maeterlinck}

Et s'il revenait un jour

Que faut-il lui dire?

- Dites-lui qu'on l'attendit

Jusqu'à en mourir...

Et s'il m'interroge encore

Sans me reconnaître?

- Parlez-lui comme une sœur,

Il souffre peut-être...

Et s'il demande où vous êtes

Que faut-il répondre?

- Donnez-lui mon anneau d'or

Sans rien lui répondre...

Et s'il veut savoir pourquoi

La salle est déserte?

- Montrez-lui la lampe éteinte

Et la porte ouverte...

Et s'il m'interroge alors

Sur la dernière heure?

- Dites-lui que j'ai souri

De peur qu'il ne pleure... ${ }^{34}$

\footnotetext{
${ }^{34}$ Extrait de Quinze chansons, 1900, II.
} 


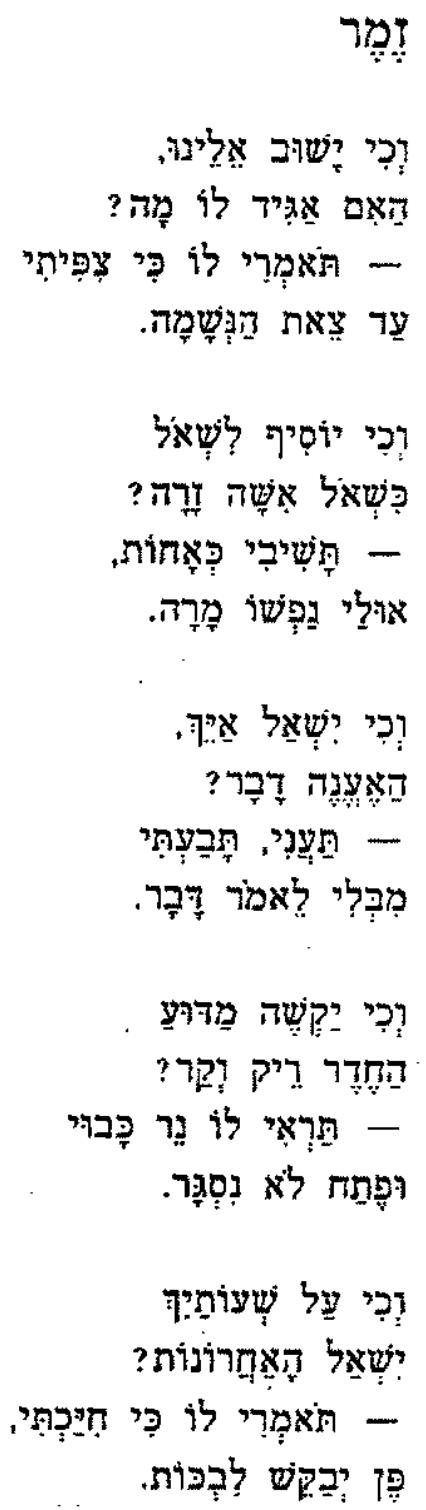




\section{3 - Charles Van Lerberghe}

Ma sœur la Pluie

Ma sœur la Pluie,

La belle et tiède pluie d'été,

Doucement vole, doucement fuit,

À travers les airs mouillés.

Tout son collier de blanches perles

Dans le ciel bleu s'est délié.

Chantez les merles,

Dansez les pies!

Parmi les branches qu'elle plie,

Dansez les fleurs, chantez les nids :

Tout ce qui vient du ciel est béni.

De ma bouche elle approche

Ses lèvres humides de fraises des bois ;

Rit, et me touche,

Partout à la fois,

De ses milliers de petits doigts.

Sur des tapis de fleurs sonores,

De l'aurore jusqu'au soir,

Et du soir jusqu'à l'aurore,

Elle pleut et pleut encore,

Autant qu'elle peut pleuvoir.

Puis, vient le soleil qui essuie,

De ses cheveux d'or,

Les pieds de la Pluie ${ }^{35}$.

${ }^{35}$ Extrait de La Chanson d’Ève, 1904, Premières paroles. 


$$
\begin{aligned}
& \text { אָחִי הַפָּטָר } \\
& \text { אָחִי הַחָּטָר, }
\end{aligned}
$$

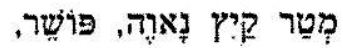

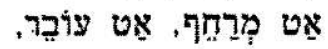

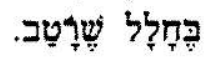

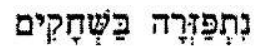

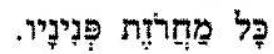

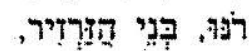

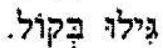

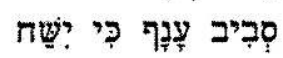

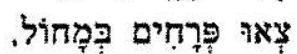

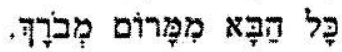

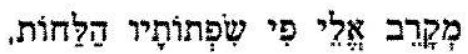

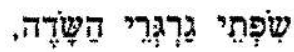

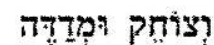

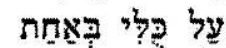

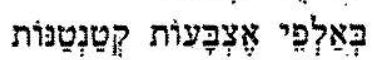

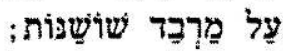

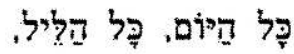

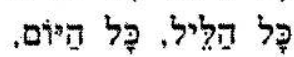

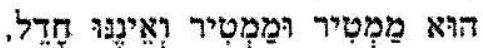

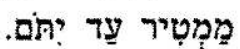

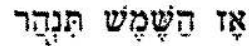

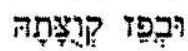

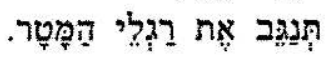


Ne suis-je vous, n'êtes-vous moi,

Ô choses que de mes doigts

Je touche et de la lumière

De mes yeux éblouis?

Fleurs où je respire, soleil où je luis,

Âme qui penses,

Qui peut me dire où je finis,

Où je commence?

Ah ! que mon cœur infiniment

Partout se retrouve! Que votre sève

C'est mon sang!

Comme un beau fleuve,

En toutes choses la même vie coule,

Et nous rêvons le même rêve ${ }^{36}$.

${ }^{36}$ Extrait de La Chanson d'Ève, Premières paroles. Ce poème se situe au début de la première partie du recueil tandis que le précédent se trouve vers la fin de celle-ci. 


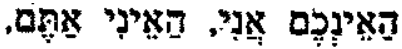

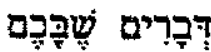

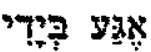

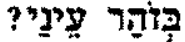

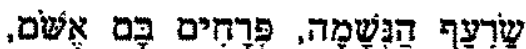

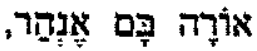

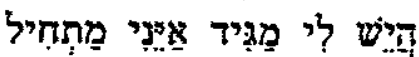

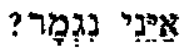

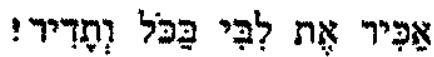

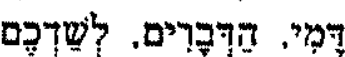

דוּא לֶדה

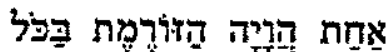

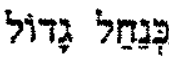

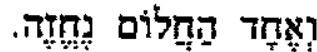


Quand vient le soir,

Des cygnes noirs,

Ou des fées sombres,

Sortent des fleurs, des choses, de nous :

Ce sont nos ombres.

Elles avancent; le jour recule.

Elles vont dans le crépuscule,

D'un mouvement glissant et lent.

Elles s'assemblent, elles s'appellent,

Se cherchent sans bruit,

Et toutes ensemble,

De leurs petites ailes,

Font la grande nuit.

Mais l'Aube dans l'eau

S'éveille et prend son grand flambeau.

Puis elle monte,

En rêve monte, et peu à peu,

Sur les ondes elle s'élève

Sa tête blonde,

Et ses yeux bleus.

Aussitôt, en fuite furtive,

Les ombres s'esquivent,

On ne sait où.

Est-ce dans l'eau ? Est-ce sous terre ?

Dans une fleur? Dans une pierre?

Est-ce dans nous?

On ne sait pas. Leurs ailes closes

Enfin reposent.

Et c'est matin ${ }^{37}$.

${ }^{37}$ Extrait de La Chanson d'Ève, La tentation. 


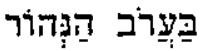

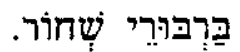

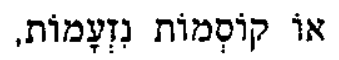

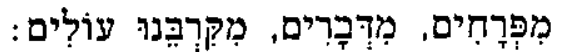

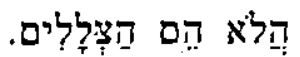

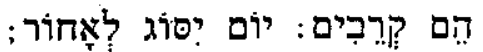

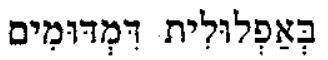

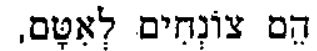

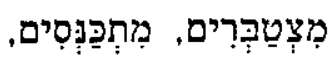

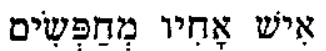

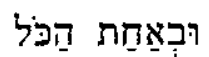

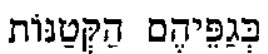

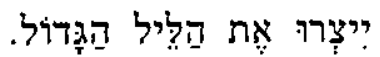

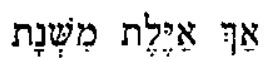

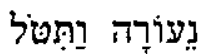

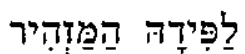

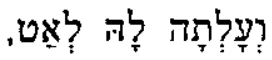

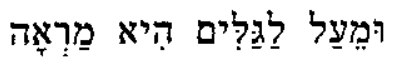

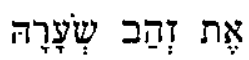

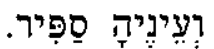

$$
\begin{aligned}
& \text { רוּמִיָּד הַצְּלָלים }
\end{aligned}
$$

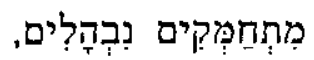

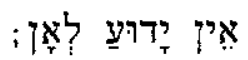

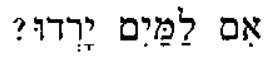

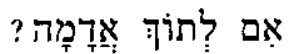

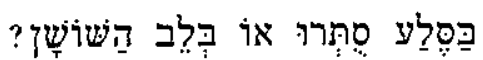

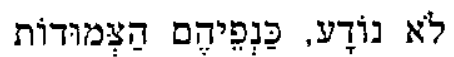

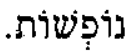

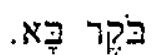




\section{Francis Jammes :}

Prière pour aller au Paradis avec les ânes

Lorsqu'il faudra aller vers vous, ô mon Dieu, faites que ce soit par un jour où la campagne en fête poudroiera. Je désire, ainsi que je fis ici-bas, choisir un chemin pour aller, comme il me plaira, au Paradis, où sont en plein jour les étoiles. Je prendrai mon bâton et sur la grande route j'irai, et je dirai aux ânes, mes amis :

Je suis Francis Jammes et je vais au Paradis, car il n'y a pas d'enfer au pays du Bon-Dieu. Je leur dirai : Venez, doux amis du ciel bleu, pauvres bêtes chéries qui, d'un brusque mouvement d'oreille, chassez les mouches plates, les coups et les abeilles...

Que je vous apparaisse au milieu de ces bêtes que j'aime tant parce qu'elles baissent la tête doucement, et s'arrêtent en joignant leurs petits pieds d'une façon bien douce et qui vous fait pitié. J'arriverai suivi de leurs milliers d'oreilles, suivi de ceux qui portèrent au flanc des corbeilles, de ceux traînant des voitures de saltimbanques ou des voitures de plumeaux et de fer-blanc, de ceux qui ont au dos des bidons bossués, des ânesses pleines comme des outres, aux pas cassés, de ceux à qui l'on met de petits pantalons à cause des plaies bleues et suintantes que font les mouches entêtées qui s'y groupent en ronds. Mon Dieu, faites qu'avec ces ânes je vous vienne. Faites que dans la paix, des anges nous conduisent vers des ruisseaux touffus où tremblent des cerises lisses comme la chair qui rit des jeunes filles, et faites que, penché dans ce séjour des âmes, sur vos divines eaux, je sois pareil aux ânes qui mireront leur humble et douce pauvreté à la limpidité de l'amour éternel ${ }^{38}$.

\footnotetext{
${ }^{38}$ Extrait de Le Deuil des primevères, 1901, Quatorze prières, VIII.
} 


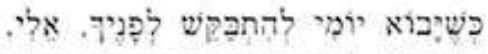

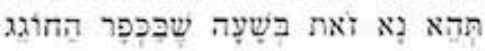

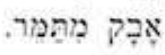

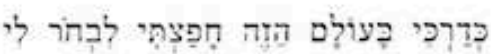

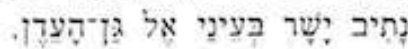

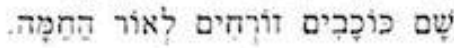

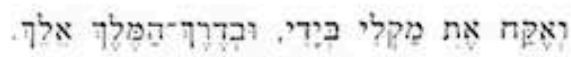

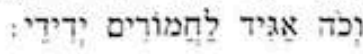

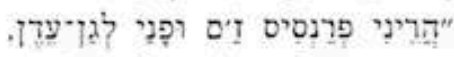

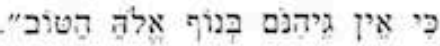

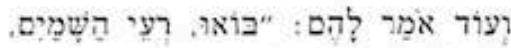

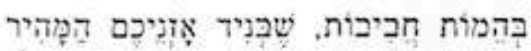

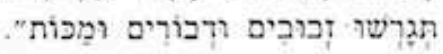

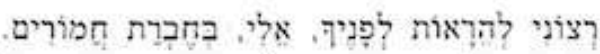

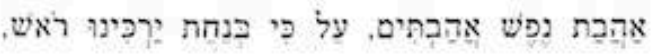

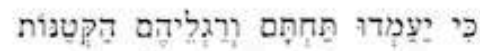

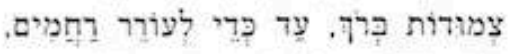

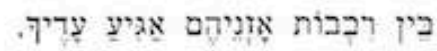

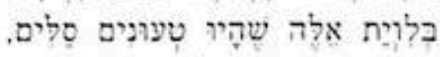

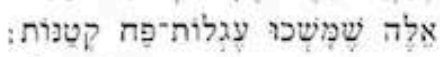

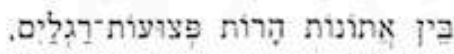

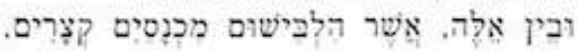

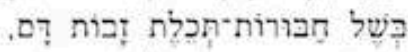

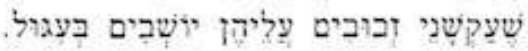

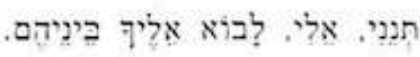

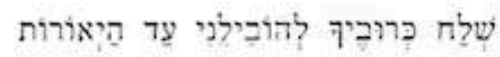

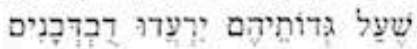

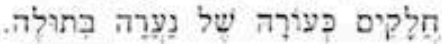

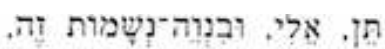

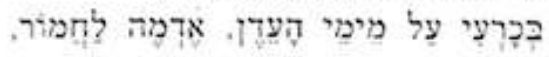

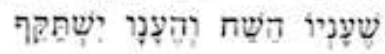

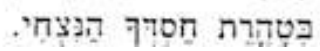


Il va neiger...

Il va neiger dans quelques jours. Je me souviens de l'an dernier. Je me souviens de mes tristesses au coin du feu. Si l'on m'avait demandé : qu'est-ce ? J'aurais dit : laissez-moi tranquille. Ce n'est rien ${ }^{39}$.

${ }^{39}$ Extrait de De l'Angélus de l'aube à l'Angélus du soir, 1898. 


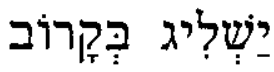

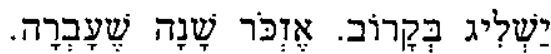

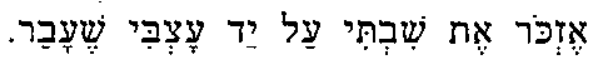

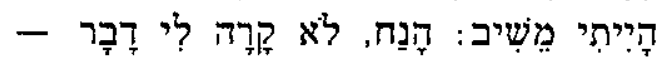

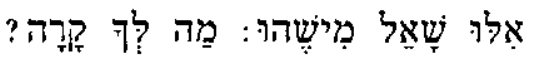


La salle à manger

Il y a une armoire à peine luisante qui a entendu les voix de mes grand'tantes, qui a entendu la voix de mon grand-père, qui a entendu la voix de mon père.

À ces souvenirs l'armoire est fidèle.

On a tort de croire qu'elle ne sait que se taire, car je cause avec elle.

Il y a aussi un coucou en bois.

Je ne sais plus pourquoi il n'a plus de voix.

Je ne veux pas le lui demander.

Peut-être qu'elle est cassée, la voix qui était dans son ressort, tout bonnement comme celle des morts.

Il y a aussi un vieux buffet qui sent la cire, la confiture, la viande, le pain et les poires mûres.

C'est un serviteur fidèle qui sait qu'il ne doit rien nous voler.

Il est venu chez moi bien des hommes et des femmes qui n'ont pas cru à ces petites âmes. Et je souris que l'on me pense seul vivant quand un visiteur me dit en entrant : - comment allez-vous, monsieur Jammes $?^{40}$

\footnotetext{
${ }^{40}$ Extrait de De l'Angélus de l'aube à l'Angélus du soir. Dans ce recueil, le présent poème se situe plutôt au début et le précédent plutôt à la fin.
} 


\section{חָחדר הָאכֶל}

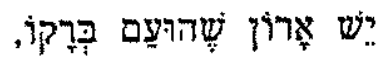

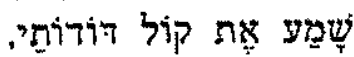

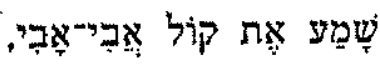

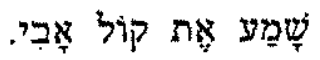

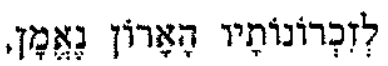

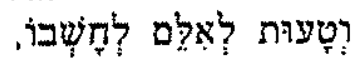

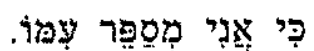

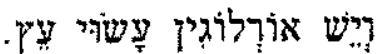

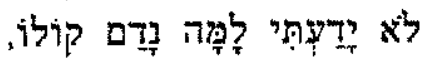

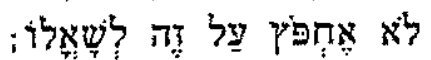

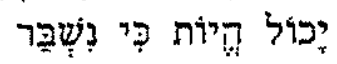

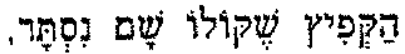

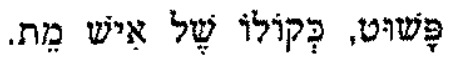

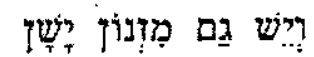

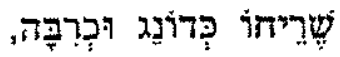

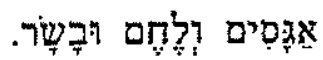

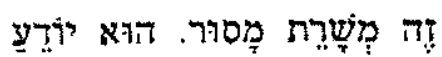

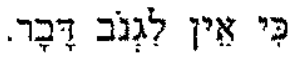

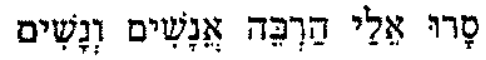

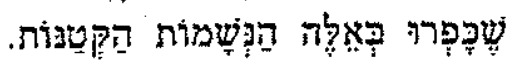

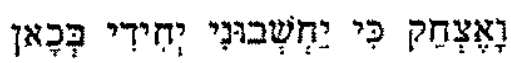

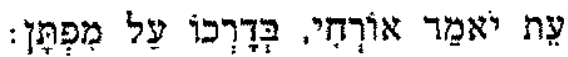

- 